\title{
Targeted next-generation sequencing identifies novel variants in candidate genes for Parkinson's disease in Black South African and Nigerian patients
}

Oluwafemi G. Oluwole ${ }^{1}$, Helena Kuivaniemi ${ }^{1}$, Shameemah Abrahams' ${ }^{1}$, William L. Haylett ${ }^{1,2}$, Alvera A. Vorster ${ }^{3}$, Carel J. van Heerden ${ }^{3}$, Colin P. Kenyon ${ }^{1,4,5,6}$, David L. Tabb ${ }^{1,4,5,6,7}$, Michael B. Fawale ${ }^{8}$, Taofiki A. Sunmonu', Abiodun Ajose ${ }^{10}$, Matthew O. Olaogun ${ }^{11}$, Anastasia C. Rossouw ${ }^{12}$, Ludo S. van Hillegondsberg ${ }^{12,13}$, Jonathan Carr ${ }^{13}$, Owen A. Ross ${ }^{14,15}$, Morenikeji A. Komolafe ${ }^{8}$, Gerard Tromp ${ }^{1,45,6,7^{*}}$ and Soraya Bardien ${ }^{1 *}$ (D)

\begin{abstract}
Background: The prevalence of Parkinson's disease (PD) is increasing in sub-Saharan Africa, but little is known about the genetics of PD in these populations. Due to their unique ancestry and diversity, sub-Saharan African populations have the potential to reveal novel insights into the pathobiology of PD. In this study, we aimed to characterise the genetic variation in known and novel PD genes in a group of Black South African and Nigerian patients.
\end{abstract}

Methods: We recruited 33 Black South African and 14 Nigerian PD patients, and screened them for sequence variants in 751 genes using an lon AmpliSeq ${ }^{\text {TM }}$ Neurological Research panel. We used bcftools to filter variants and annovar software for the annotation. Rare variants were prioritised using MetaLR and MetaSVM prediction scores. The effect of a variant on ATP13A2's protein structure was investigated by molecular modelling.

Results: We identified 14,655 rare variants with a minor allele frequency $\leq 0.01$, which included 2448 missense variants. Notably, no common pathogenic mutations were identified in these patients. Also, none of the known PD-associated mutations were found highlighting the need for more studies in African populations. Altogether, 54 rare variants in 42 genes were considered deleterious and were prioritized, based on MetaLR and MetaSVM scores, for follow-up studies. Protein modelling showed that the S1004R variant in ATP13A2 possibly alters the conformation of the protein.

Conclusions: We identified several rare variants predicted to be deleterious in sub-Saharan Africa PD patients; however, further studies are required to determine the biological effects of these variants and their possible role in PD. Studies such as these are important to elucidate the genetic aetiology of this disorder in patients of African ancestry.

Keywords: Parkinson's disease, Next-generation sequencing, Scoring of sequence variants, Sub-Saharan Africa, South Africa, Nigeria, Sequence variants

\footnotetext{
* Correspondence: gctromp@sun.ac.za; sbardien@sun.ac.za

${ }^{1}$ Division of Molecular Biology and Human Genetics, Department of

Biomedical Sciences, Stellenbosch University, Cape Town, South Africa

Full list of author information is available at the end of the article
}

(c) The Author(s). 2020 Open Access This article is distributed under the terms of the Creative Commons Attribution 4.0 International License (http://creativecommons.org/licenses/by/4.0/), which permits unrestricted use, distribution, and reproduction in any medium, provided you give appropriate credit to the original author(s) and the source, provide a link to the Creative Commons license, and indicate if changes were made. The Creative Commons Public Domain Dedication waiver (http://creativecommons.org/publicdomain/zero/1.0/) applies to the data made available in this article, unless otherwise stated. 


\section{Background}

Parkinson's disease (PD) is a debilitating neurodegenerative disorder that impairs patients' motor skills, and speech coordination. It is one of the leading causes of disability and mortality among neurological disorders globally [1]. The neuropathological hallmark of PD is the progressive loss of predominantly dopaminergic neurons of the substantia nigra pars compacta of the midbrain, which regulate voluntary movement. The diagnosis of this disorder is largely clinical using criteria such as the UK PD Society Brain Bank criteria (UKPDSBBC) to differentiate ageing related symptoms from PD [2]. The pathobiology is yet to be fully elucidated, but environmental and genetic factors have been linked to PD aetiology [3, 4]. PD symptoms usually manifest in the same way in all patients, but the prevalence, incidence and risk factors may vary according to the geographical region [5]. Estimates of PD prevalence in sub-Saharan Africa (SSA) vary widely across previous studies and range from 10 to $235 / 100,000$ in urban populations $[6,7]$.

Genetics as an etiologic concept in PD has been wellestablished [8, 9]. Approximately $5-10 \%$ of PD patients have a familial form of the disease, which is due to highly penetrant, rare pathogenic mutations [9]. For sporadic forms of this disorder, the genetics is complex as common genetic variants may act in concert with environmental factors [9-11]. The genetic discoveries have led to important hypotheses about the mechanisms underlying PD, which include dysfunction of the ubiquitin-proteasome system and mitochondrial dysfunction coupled with oxidative stress [12].

Most of the studies on the established PD genes or genes associated with $\mathrm{PD}$ including SNCA, LRRK2, PRKN, PINK1, PARK7, ATP13A2 and GBA, have been performed in European, North American, North African Arab or Asian populations [9, 13, 14]. In general, limited studies exist on the genetics of PD in the Black African populations [15]. It has been suggested that the variants most commonly associated with $\mathrm{PD}$ are rare among South African PD patients [16, 17]. Similarly, a previous genetic study screened for mutations in LRRK2, PRKN and ATXN3 in 57 Nigerian PD patients but did not identify any pathogenic mutations [18]. African populations have a diverse ancestry, and have more private alleles than any other population, suggesting that the genetic aetiology of PD in African populations could be unique [19].

Next-Generation Sequencing (NGS) provides a way to explore the genetic basis of diseases, and has resulted in the discovery of a large number of disease-associated mutations [20]. In contrast to whole-genome or wholeexome sequencing [21, 22], targeted sequencing panels [23] focus the analysis on specific genes of interest. The
Ion AmpliSeq ${ }^{\mathrm{Tm}}$ Neurological Research Panel is a commercially available panel designed to screen genes linked to neurological disorders as well as genes involved in brain function. The primary goals of the present study were to use this panel to determine whether a common pathogenic mutation was present, and to characterise the genetic variation in known and novel PD genes, in a group of Black South African and Nigerian PD patients.

\section{Methods}

\section{Study participants}

The study group consisted of 33 unrelated Black South African PD patients and 14 unrelated Nigerian PD patients. South African patients were primarily recruited at the Neurology Clinic of Tygerberg Academic Hospital, Cape Town, South Africa, and at the Neurology Clinic of Frere Hospital, East London, South Africa. Nigerian PD patients were recruited at the Neurology Clinic, Obafemi Awolowo University Teaching Hospitals Complex, Ile-Ife, Nigeria. All patients were confirmed to have PD by neurologists, based on the UKPDSBBC diagnostic criteria. All patients provided written informed consent to take part in the study and provided peripheral blood samples for genetic studies. This study was approved by the Health Research Ethics Committee of Stellenbosch University (HREC 2002/C059, N16/04/041 and S16/08/ 151), and the Ethics and Research Committee of Obafemi Awolowo University Teaching Hospitals (ERC/ 2015/08/15). Demographic information and clinical characteristics of the patients are provided in Table 1 and Additional file 1: Table S1.

\section{Quality control and annotation of targeted next- generation sequencing (tNGS) data}

The Ion AmpliSeq ${ }^{\mathrm{Tm}}$ Neurological Research panel and the Ion AmpliSeq ${ }^{\text {тM }}$ Library Kit 2.0 (Thermo Scientific, Waltham, Massachusetts, USA) were used for multiplex PCR amplification of 751 genes (Additional file 2: Table S2). The intronic regions incorporated as part of the exon targets are listed separately in Additional file 3: Table S3. Details on the library construction and next-generation-sequencing protocols are available in Additional file 4.

The flow space calibration, base calling, alignment with the reference genome (GRCh38-hg19), coverage analysis and variant calling were performed using standard parameters in the Ion Torrent Software Suite (ISS) version 5.4.0. Sequenced variants, including insertions and deletions (INDELs), splice site variants, single nucleotide variants (SNVs), multiple nucleotide variants (MNVs), as well as variants in the $3^{\prime}$ untranslated region (UTR3) and 5' untranslated region (UTR5) were identified. The variant call format (VCF) files produced by the ISS were filtered using bcftools to ensure that: 
Table 1 Characteristics of the 47 Parkinson's disease patients

\begin{tabular}{lll}
\hline Characteristic & Black South African & Nigerian \\
& $N=33$ & $N=14$ \\
\hline Sex, male, $n(\%)$ & $18(54)$ & $11(78)$ \\
Average age-at-onset \pm SD (range), years & $48 \pm 8(30-59)$ & $63 \pm 13(36-80)$ \\
Average age at recruitment \pm SD (range), years & $55 \pm 11(35-78)$ & $67 \pm 11(42-81)$ \\
Positive family history of PD, $n(\%)$ & $2^{\text {a }(6)}$ & 0 \\
\hline
\end{tabular}

${ }^{a}$ Individuals s43_059 and s94_069 have a possible Mendelian inheritance pattern for PD

FILTER $=$ PASS (the ISS internal criteria for setting the PASS filter were met).

QUAL > 100 (the quality score exceeded 100).

$\mathrm{FMT} / \mathrm{AO} \geq 20$ (there were at least 20 reads for the alternative allele).

$\mathrm{FMT} / \mathrm{DP} \geq 40$ (there were at least 40 reads in total).

$\mathrm{FMT} / \mathrm{SAF} \geq 5$ (there were at least 5 reads of the alternative allele in the forward direction).

$\mathrm{FMT} / \mathrm{SAR} \geq 5$ (there were at least 5 reads of the alternative allele in the reverse direction).

These criteria ensured that the observation was made in both directions with at least five reads in either direction, the overall depth was at least 40 and the alternative allele depth was at least 20 .

The sequencing data on the 47 samples were of good overall quality. We plotted a graph for the target region coverage using the bam files generated by the Ion Torrent Variant Caller (Additional file 5: Figure S1). This graph showed that 41 samples had at least $80 \%$ coverage of the target region at an average read depth of 40X, three samples had a coverage of $78-79 \%$, and another three samples had a coverage of $61-76 \%$.

The VCF files were merged into a single file and processed with the utility annovar (annovar.openbioinformatics.org/) to produce an annotation file for all the variants that passed the criteria above. All available annotations were included. These included conservation scores, allele frequencies and functional predictions (Additional file 6: Table S4). Perl (https:// www.perl.org/) was used to extract data and analyses were carried out in R (R Core Team, 2018) [24]. Variants were extracted as homozygous or heterozygous for the alternative allele. The quality scores for all the variants passing the filters were extracted and analysed in R. Using the bam files, bedtools was used to generate statistics on the coverage (depth of sequencing) for the regions in the Ampliseq capture panel as defined by the manufacturer's bed file. We focused on variants that are rare in control populations as defined by a minor allele frequency (MAF) threshold of 0.01 . We created global classifications of variants and generated a summary of variant types, to encode variants as synonymous, missense or frameshift in the variable amino acid class, insertion, deletion or substitution in the variable mutation type, as well as single or multiple base variants in the variable mutated base. We merged the variant summary (whether the variant was observed as a homozygous or heterozygous), with the annotation.

\section{Variant prioritisation}

We used the prediction scores MetaLR and MetaSVM for selecting deleterious sequence variants (Additional file 6: Table S4). MetaLR and MetaSVM are themselves ensembles (composite models) of many other scores [25]. Currently, these two have the best performance on curated data sets (training and test) of non-synonymous variants that contain both deleterious (protein-function altering) and benign variants. We therefore used these to prioritize the rare variants in our data. Both metrics were scaled as probabilities $[0,1]$ with scores close to 1 indicating certainty that the variant is deleterious. We used a score of $>0.8$ as a cut-off for including the variant into our list of rare "pathogenic variants" as recommended by Liu et al [25]. We also used 24 other variant scoring algorithms. We plotted the correlation matrix of all 26 scoring algorithm outputs used in our study (Additional file 7: Figure S2). We generated Radar plots (http://www.cmap.polytechnique.fr/ lepennec/ $\mathrm{R} /$ Radar/RadarAndParallelPlots.html) for each variant likely to be deleterious to demonstrate the correlation among 17 different scoring algorithms. All scores were standardized to $0-1$ scale with score 1 (furthest from the centre of the graph) indicating strongest evidence that the variant is deleterious.

\section{Protein structures and modelling for functional prediction}

To determine the consequences introduced by potential pathogenic variants on the protein structure, we selected a variant in $A T P 13 A 2$, for this analysis. We extracted protein information from the Protein Data Bank (PDB) of the Research Collaboratory for Structural Bioinformatics (RCSB) (https://www.rcsb.org/) [26]. The structure of ATP13A2 was modelled by submitting the 1180 amino acid UniProt accession ACQ9NQ11 to the Phyre2 server [27]. Phyre2 is a suite of tools available on the web to predict and analyse protein structure, function and mutations. The predicted ATP13A2 structure conformed well with those of known P-type ATPase cation pumps [28-31]. This structure was 
used for additional modelling using the Maestro 11.4 suite of software (Schrödinger Inc., Cambridge, MA). The protein parameterization was carried out using the Maestro Preparation Wizard. The $\mathrm{Ca}^{2+}$ binding sites were identified based on the availability of coordinating glutamate, aspartate, asparagine and glutamine sidechains as found in the other P-type ATPase structures. The two $\mathrm{Ca}^{2+}$ ions were manually docked into the active sites and the structure's energy minimized. The S1004R mutation was generated using in silico mutagenesis with subsequent energy minimization. Based on these structures, the interaction network between the mutated site and $\mathrm{Ca}^{2+}$ was identified (Kenyon et al. unpublished results).

\section{Results}

The mean age-at-onset (AAO) of PD in patients varied between the two study groups. It was $48 \pm 8$ years and $63 \pm 13$ years for the South African and Nigerian patients, respectively (Table 1 ). This may be because recruitment in South Africa was predominantly focussed on patients with earlier AAO ( $<50$ years). Two of the South African PD patients had a positive family history with both having an affected sibling and an affected parent.

\section{Identification of sequence variants}

We applied stringent threshold criteria for the filtering and annotation of the variants to exclude low quality variants. Altogether 25,917 sequence variants passed quality control. We then removed all variants with MAF $>0.01$ in any of the sequencing databases used as reference databases for the study (Additional file 6: Table S4) and were left with 14,655 rare variants. These rare variants could be classified into 7934 intronic and 5695 exonic variants (Fig. 1; an interactive html-version of the figure is at BMC website). They included 198 UTR5 and 341 UTR3 variants, as well as 32 frameshift, 3175 synonymous and 2448 missense variants. Altogether 14,057 were SNVs and 598 MNVs. There were 261 insertions, 600 deletions and 13,794 substitutions.

In addition, we separately screened 16 PD genes on the panel (SNCA, LRRK2, PRKN, PINK1, PARK7, ATP13A2, EIF4G1, GIGYF2, PLA2G6, FBXO7, VPS35, MAPT, HTRA2, SPG11, GRN and DCTN1) for all sequence variants, and these results are shown in Additional file 8: Table S5).

\section{Pathogenicity prediction of variants}

To determine which rare variants are likely to be deleterious and could potentially contribute to the PD

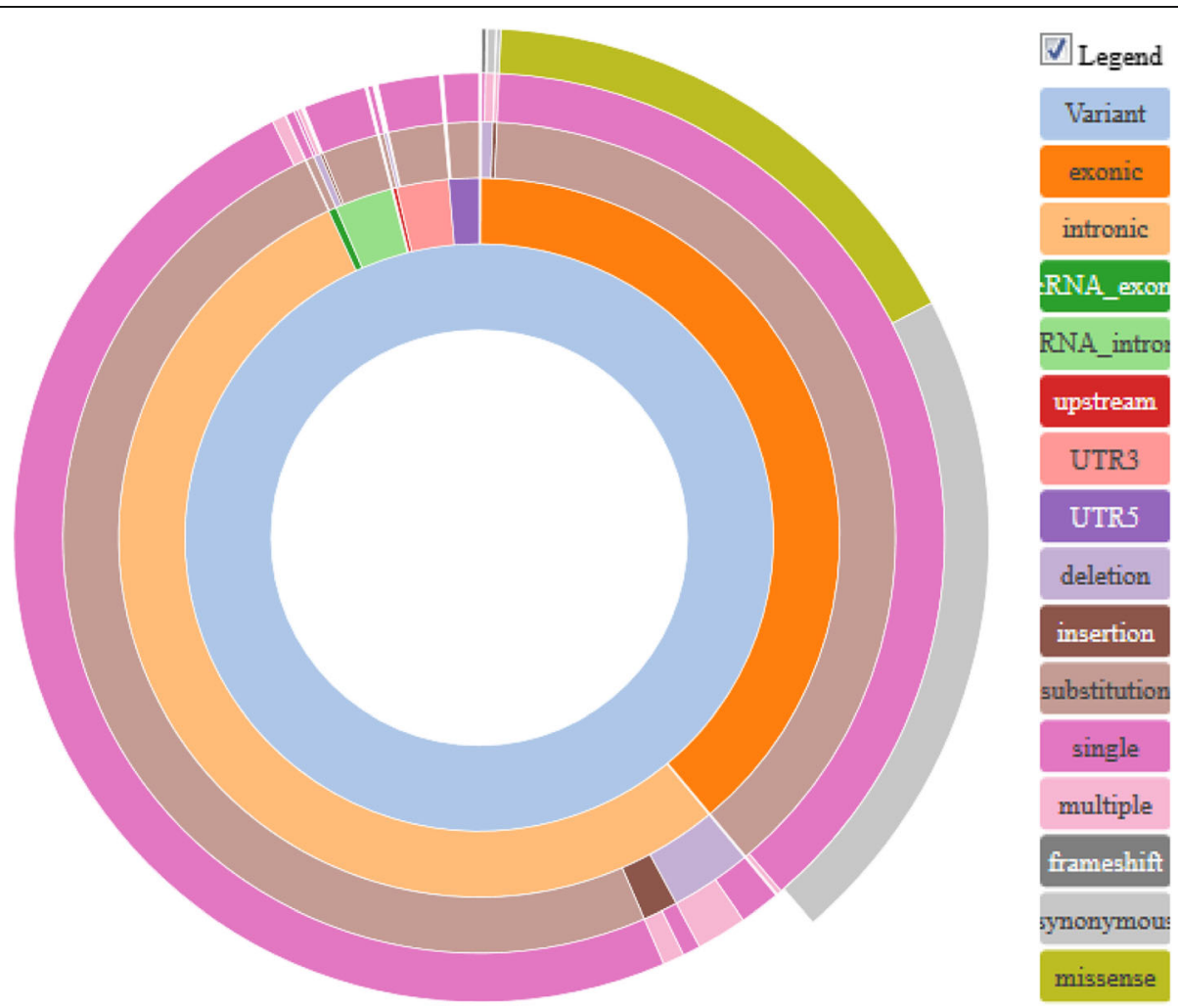

Fig. 1 Sunburst diagram showing the functional classes of 14,655 rare (MAF $\leq 0.01$ ) sequence variants identified in 33 Black South African and 14 Nigerian PD patients. An interactive HTML-version of the figure is available at BMC website 
pathobiology in the study participants, we used MetaLR and MetaSVM. We focused on identifying rare (MAF $\leq$ 0.01 ) or novel (not seen in any of the databases listed in Additional file 6: Table S4) exonic variants predicted to be deleterious. The goal was to minimize the number of false positives by applying stringent filtering criteria. Altogether, 52 heterozygous, one hemizygous and one homozygous exonic rare (MAF $\leq 0.01)$ missense variants predicted to be deleterious were found in 42 genes (Tables 2 and 3). This included a heterozygous missense variant in one of the known PD genes, ATP13A2 $(S 1004 R)$ which was validated by Sanger sequencing (data not shown). Radar plots demonstrating pathogenicity scores for each of these 54 rare variants are shown in Additional file 9: Figure S3.

We then analysed separately the two South African individuals (S43_059 and S94_069) with a positive family history of PD (one affected sibling and an affected parent). They each had three heterozygous variants (Table 3). Both individuals had a pyruvate carboxylase $(P C)$ R732G variant (rs112948607). Additionally, individual S43_059 carried galactosylceramidase (GALC) T445S (rs34134328) and TSC complex subunit 2 (TSC2) S1092 L (rs148527903) variants; while S94_069 carried arylsulfatase A (ARSA) N442S (rs6151427) and prickle planar cell polarity protein 2 (PRICKLE2) Q274P (rs564701683) variants. All of the variants had a high certainty of being predicted to be deleterious (pathogenicity score $>0.8$ ) (Table 2). The GALC variant was excluded based on its high MAF in GnomAD African controls $(\mathrm{MAF}=0.016)$ but all of the other variants are rare $(\mathrm{MAF}<0.01)([32]$; Table 2) and are therefore potential candidates. Notably, the $P C$ R732G variant that they both share was not found in any of the other patients screened. However, since there was only DNA available for one affected sibling for each of these patients, co-segregation analysis of the variants with disease could not be performed.

We attempted to prioritise one possible pathogenic variant per patient based on MAF $(<0.01)$, pathogenicity prediction scores $(>0.8)$ and evidence of prior association of the gene/protein with PD or Parkinsonism (Table 2; Additional file 10: Table S6). In some cases, the MAF of the variant in African controls in GnomAD was $\geq 0.01$, similar to the frequency observed in the patients (Table 2), and those variants were therefore excluded. The prioritised variants are shown in bold and in green font in Table 3. In a few individuals, one variant could not be prioritised over others as more than one variant fulfilled these criteria.

\section{Protein modelling for the S1004R variant in ATP13A2}

When the S1004R variant was inserted into the ATP13A2 structure and energy minimized (Kenyon et al. unpublished results), we found that the peptide backbone around the cation binding site was displaced, changing the distance between the cation and a coordinating atom from 3.44 to $2.66 \AA$ (Additional file 11: Figure S4). It could be postulated that the conformational change may alter the efficiency of the pump by interfering with the reaction cycle [28-30].

\section{Discussion}

In this study, 47 Black South African and Nigerian PD patients were screened and 54 potentially deleterious sequence variants with $\mathrm{MAF} \leq 0.01$ in 42 different genes were identified. The 751-gene panel used in the current study contains only 16 of the 34 known PD genes, but it does have six genes (ATP13A2, LRRK2, PARK7, PINK1, $P R K N$, and $S N C A$ ) with strong prior evidence of being involved in PD pathobiology. We identified a rare sequence variant predicted to be deleterious in only one of these genes, ATP13A2. Notably, we did not identify any of the previously reported pathogenic PD mutations catalogued in the PDmutDB database (https://www.molgen. vib-ua.be/PDMutDB/database) in the SSA patients. One possible reason is that, as seen in previous genetic studies on SSA PD patients, common mutations such as LRRK2 G2019S may be a rare cause of PD in these populations [15].

Protein modelling analysis of the ATP13A2 S1004R variant, which was found in a South African patient (AAO of 39 years), revealed that the variant is potentially functionally important. An interaction between R1004 and the cation binding site was identified suggesting that the variant would interfere with the function of ATP13A2 as a pump of inorganic cations such as metal ions. A previous functional study demonstrated that increased expression of ATP13A2 supresses $\alpha$-synuclein toxicity in neural cells and that ATP13A2 was likely to act as a $\mathrm{Zn}^{2+}$ pump [33]. Thus, this variant could potentially contribute to PD however, wet-laboratory functional studies are necessary to prove that the variant is indeed pathogenic.

In the two patients with a possible Mendelian inheritance of PD, five heterozygous variants were identified. Co-segregation analysis of the variants with disease in these families was not possible due to a lack of DNA of the family members. However, none of the genes in which these variants were found has been linked to Mendelian forms of PD. In fact, mutations within the $P C, P R I C K L E 2$ and TSC2 genes have previously been associated with non-neurodegenerative diseases including diseases involved in energy deficiency, tumour formation and seizures [34-36]. Therefore, it is unlikely that mutations in these genes would contribute to a Mendelian inheritance pattern of $\mathrm{PD}$ in these patients. ARSA mutations, similar to $G B A$ mutations, have been previously linked to lysosomal storage diseases (LSDs) [37]. 


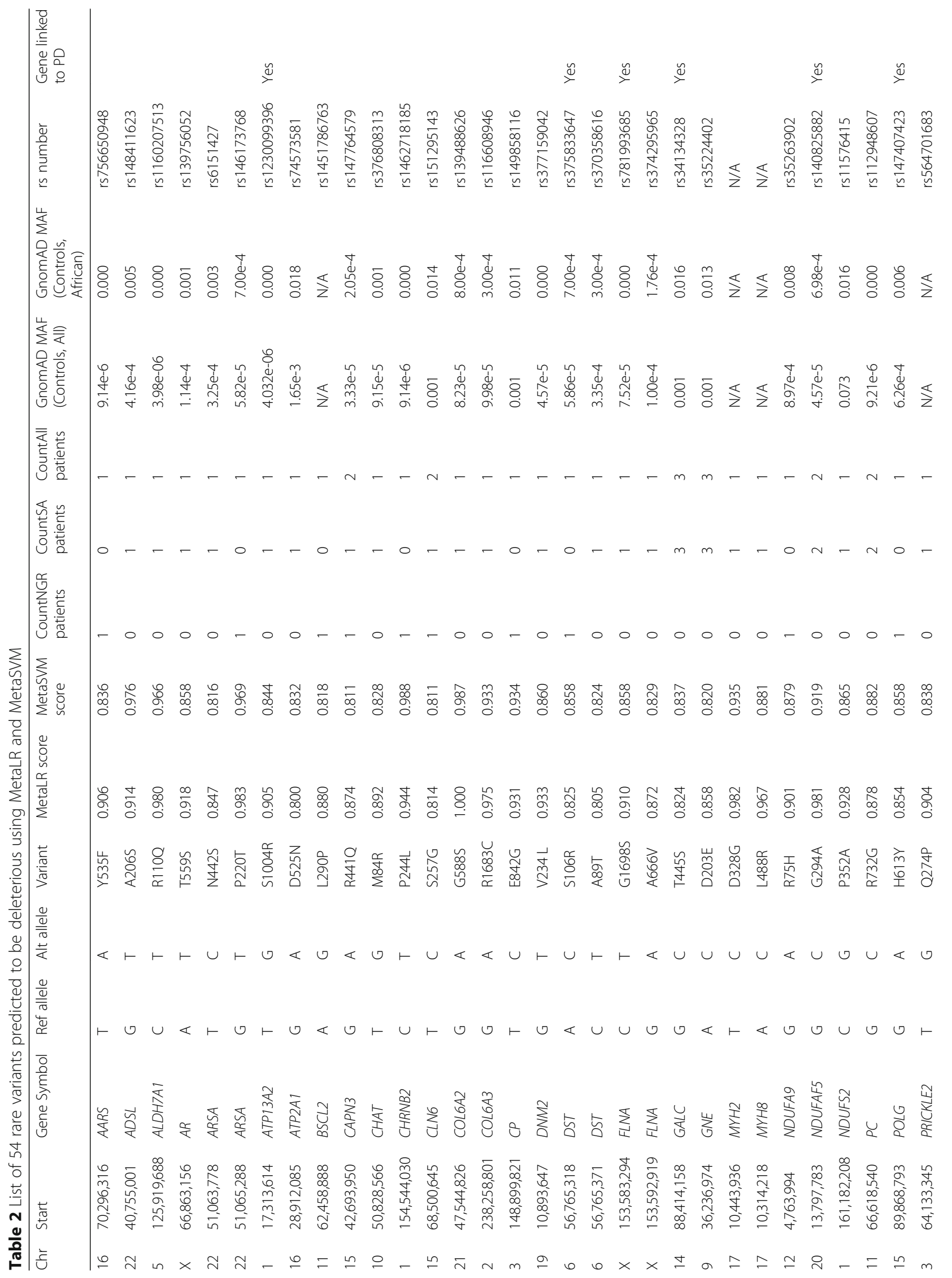




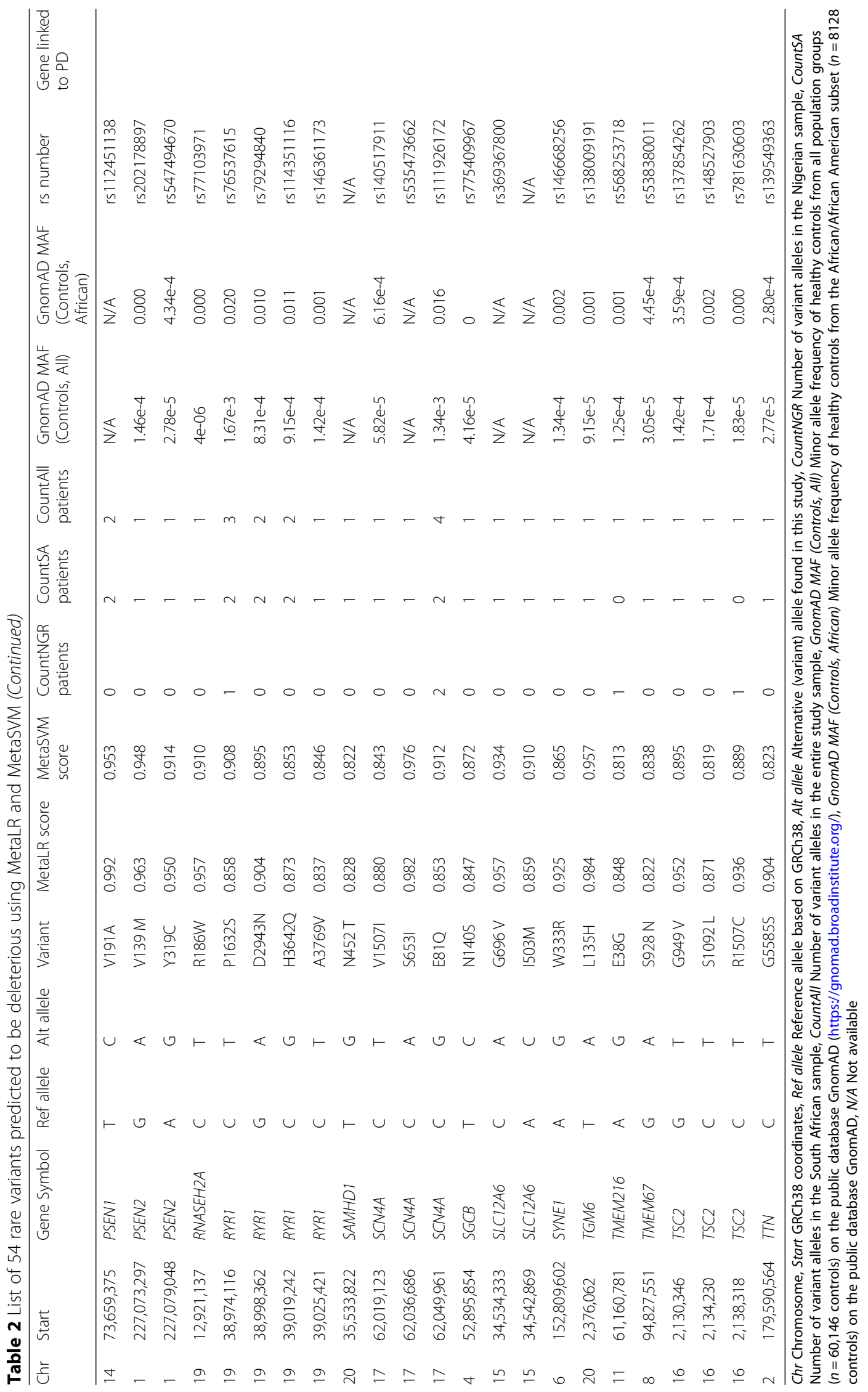




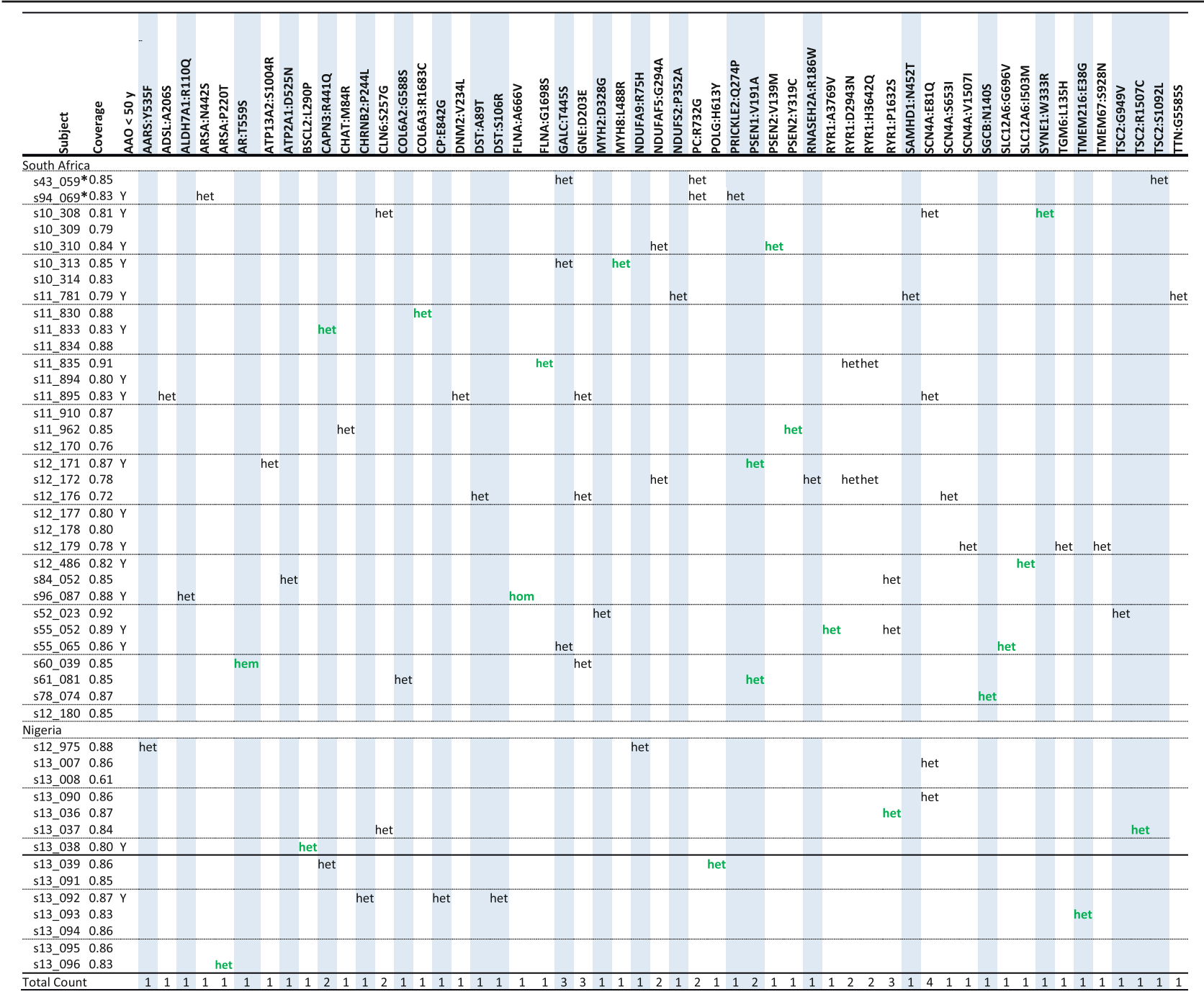

Subject, sample code; Coverage, global tNGS data coverage for the listed sample; AAO < 50 y, cases with early-onset PD are indicated (Y, yes); Other column headers indicate gene and variant for which data are provided. Hem, patient was hemizygous for the variant; het, patient was heterozygous for the variant; hom, patient was homozygous for the variant. TOTAL COUNT, number of each rare deleterious variant in the study population. *, These individuals have a positive family history of PD. The variants in bold and green font are the candidate variants prioritized for further analyses

Although lysosomal mechanisms are increasingly being shown to be important in PD pathogenesis, the interplay between genetic mutations, lysosomal storage biology and PD is complex and require further elucidation to understand the underlying biology connecting lysosomal storage and PD. However, there is no evidence currently indicating that ARSA mutations cause familial forms of PD.

Limitations of our study include the fact that the sample size was small making it difficult to estimate the actual contribution of genetic factors to PD in the SSA populations. The belief among Black SSA populations that PD is caused by witchcraft and does not have a genetic link [38] and the notion that it is part of normal ageing, may have contributed to the difficulty in recruiting more patients for the study. Also, 18 of the 34 previously identified PD genes were not on the panel. In addition, annotation of sequence variants in terms of effect on the protein using bioinformatic tools remains problematic. We chose to use the MetaLR and MetaSVM algorithms that currently appear to perform best, but functional studies are needed for validation of these results. Future studies will involve using a custom-panel that captures all of the known PD genes; using whole-exome or whole-genome sequencing; screening of the patients for copy number variations especially in the PINK1 and PRKN genes; and recruitment of a large number of ethnic-matched controls to determine the frequency of prioritized variants in these populations. 


\section{Conclusions}

Studies are urgently needed to characterise the genetic variation in the known as well as novel PD genes in the understudied SSA populations. Multi-national collaborations across Africa are essential to recruit the large numbers of patients and controls required. The current study provides a starting point to address this need and although it is acknowledged that the sample sizes used here are relatively small, the use of NGS technologies means that the full spectrum of sequence variation in 751 genes has been captured and is available for future studies. We identified several rare variants predicted to be deleterious and they provide new putative candidates for PD but further studies are required to assess their role in PD pathobiology. It is important to include SSA populations in PD genetic studies to ensure that they do not miss out on the potential benefits and opportunities promised by precision medicine [39].

\section{Supplementary information}

Supplementary information accompanies this paper at https://doi.org/10. 1186/s12881-020-0953-1.

\section{Additional file 1: Table S1. Individual clinical data on study} participants.

Additional file 2: Table S2. Neurological Research panel information provided by Ion AmpliSeq ${ }^{\mathrm{TM}}$ (WWw.ampliseq.com)

Additional file 3: Table S3. Intron regions covered as part of the exon targets.

Additional file 4. Protocols for library construction and Ion Torrent sequencing.

Additional file 5: Figure S1. Target region coverage for the 47 samples.

Additional file 6: Table S4. Tools and databases used for the annotation of sequence variants.

Additional file 7: Figure S2. Correlation between prediction and conservation scores.

Additional file 8: Table S5. Sequence variants found in the known PD genes.

Additional file 9: Figure S3. Radar plots for 54 selected rare variants.

Additional file 10: Table S6. Candidate genes with a link to Parkinson's disease or Parkinsonism.

Additional file 11: Figure S4. Stereoscopic crystal structure models of ATP13A2 showing the position of S1004R.

\footnotetext{
Abbreviations

AAO: Age at onset; ATP13A2: ATPase cation transporting 13A2; ATXN3: Ataxin 3; BLAST: Basic local alignment search tool; GBA: Betaglucocerebrosidase; HMM: Hidden markov model; INDEL: Insertion and deletion; ISS: Ion Torrent software suite; LRRK2: Leucine-rich repeat kinase 2; MAF: Minor allele frequency; MNV: Multiple nucleotide variant; NGS: Nextgeneration sequencing; PARK7: Parkinsonism associated deglycase; PD: Parkinson's disease; PDmutDB: Parkinson's disease mutation database; PINK1: PTEN induced putative kinase 1; PRKN: Parkin RBR E3 ubiquitin protein ligase; SNCA: Alpha-synuclein; SNV: Single nucleotide variant; SSA: SubSaharan Africa; tNGS: Targeted NGS; UKPDSBBC: UK PD society brain bank criteria; UTR3: 3' untranslated region; UTR5: 5' untranslated region; VCF: Variant call format
}

\section{Acknowledgements}

We thank the study participants and the recruitment staff in the Division of Neurology at Tygerberg Academic Hospital, Cape Town, and in the Division of Neurology, Department of Medicine, Faculty of Health Sciences, Walter Sisulu University, East London, in South Africa, as well as the Obafemi Awolowo University Teaching Hospitals Complex, Ile-lfe, in Nigeria. We thank Alexandra I. Beasley for assistance with Additional file 8: Table S5.

\section{Authors' contributions}

OGO performed this research to fulfil the requirements for his doctoral degree, and he obtained ethics approvals for the study, did literature searches, carried out Sanger sequencing, performed data analyses using lon Torrent software, prepared some of the tables and figures and drafted the manuscript. HK supervised the project, critically appraised the results in this study and edited the manuscript. GT co-supervised the project, performed data analysis, wrote results, generated figures and tables, and contributed to knowledge. CPK carried out some of the protein modelling. AAV and CJvH performed the tNGS, and CVJH produced one of the tables. SB and MAK conceptualised the idea for this research, obtained ethics approvals, invited other collaborators, obtained funding, supervised part of the work, contributed to knowledge, and revised the manuscript. JC led the clinical team in South Africa, obtained ethics approvals, provided clinical expertise, contributed to knowledge, and revised the manuscript. MBF, TAS, and MAK recruited patients in Nigeria, provided clinical expertise and revised the manuscript. ACR, and $\mathrm{LSVH}$ recruited patients in South Africa, provided clinical expertise and revised the manuscript. AA, MOO, OAR and DLT revised the manuscript and contributed to knowledge, and OAR produced one of the tables. SA and WLH assisted with critical reworking and revising of the final version of the manuscript. All authors approved the final version of the manuscript.

\section{Funding}

This work was supported by a grant (R21NS098862) from the National Institute of Neurological Disorders and Stroke, and Fogarty International Center, the National Institutes of Health, USA, Grant 106052 from the South African National Research Foundation, and a Self-Initiated Research Grant from the South African Medical Research Council to SB. HK was supported by the Faculty of Medicine and Health Sciences, Stellenbosch University, South Africa. JC received support from the South African National Research Foundation. SA was supported by the NRF Innovation Postdoctoral Fellowship from the South African National Research Foundation. GT and DLT were supported by the South African Tuberculosis Bioinformatics Initiative (SATBBI), a Strategic Health Innovation Partnership grant from the South African Medical Research Council and South African Department of Science and Technology. This work was also supported by an NIH grant (P50NS072187 to OAR) for the Mayo Clinic Morris K. Udall Center of Excellence in Parkinson's Disease Research Lewy Body Dementia Association (LBDA) Research Center of Excellence, and an American Parkinson Disease Association (APDA) Center for Advanced Research grant to OAR.

The funding bodies played no role in the design of the study and collection, analysis, and interpretation of data and in writing the manuscript.

\section{Availability of data and materials}

The bam files of the tNGS data have been deposited to the European Nucleotide Archive (ENA) and can be retrieved with an accession number PRJEB30330 from https://www.ebi.ac.uk/ena/browse/data-retrieval-rest.

\section{Ethics approval and consent to participate}

All patients provided written informed consent to take part in the study. This study was submitted to and approved by the Health Research Ethics Committee of Stellenbosch University in South Africa (Approval reference numbers HREC 2002/C059, N16/04/041 and S16/08/151), as well as the Ethics and Research Committee of Obafemi Awolowo University Teaching Hospitals in Nigeria (Approval reference number ERC/2015/08/15).

Consent for publication

Not applicable.

Competing interests

The authors declare that they have no competing interests. 


\section{Author details}

'Division of Molecular Biology and Human Genetics, Department of Biomedical Sciences, Stellenbosch University, Cape Town, South Africa. ${ }^{2}$ Division of Endocrinology, Department of Medicine, Faculty of Medicine and Health Sciences, Stellenbosch University, Cape Town, South Africa. ${ }^{3}$ DNA Sequencing Unit, Central Analytical Facility, Stellenbosch University, Stellenbosch, South Africa. ${ }^{4}$ Bioinformatics Unit, South African Tuberculosis Bioinformatics Initiative, Stellenbosch University, Cape Town, South Africa. ${ }^{5}$ DST-NRF Centre of Excellence for Biomedical Tuberculosis Research, Stellenbosch University, Cape Town, South Africa. ${ }^{6}$ South African Medical Research Council Centre for Tuberculosis Research, Stellenbosch University, Cape Town, South Africa. ${ }^{7}$ Centre for Bioinformatics and Computational Biology, Stellenbosch University, Stellenbosch, South Africa. ${ }^{8}$ Neurology Unit, Department of Medicine, College of Health Sciences, Obafemi Awolowo University, Ile-lfe, Nigeria. ${ }^{9}$ Neurology Unit, Department of Medicine, Federal Medical Centre, Owo, Nigeria. ${ }^{10}$ Department of Chemical Pathology, College of Health Sciences, Obafemi Awolowo University, lle-lfe, Nigeria.

${ }^{11}$ Department of Medical Rehabilitation, College of Health Sciences, Obafemi Awolowo University, lle-lfe, Nigeria. ${ }^{12}$ Division of Neurology, Department of Medicine, Faculty of Health Sciences, Walter Sisulu University, East London, South Africa. ${ }^{13}$ Division of Neurology, Department of Medicine, Faculty of Medicine and Health Sciences, Stellenbosch University, Cape Town, South Africa. ${ }^{14}$ Department of Neuroscience, Mayo Clinic, Jacksonville, Florida, USA. ${ }^{15}$ Department of Clinical Genomics, Mayo Clinic College of Medicine, Jacksonville, Florida, USA.

Received: 5 March 2019 Accepted: 10 January 2020

Published online: 04 February 2020

\section{References}

1. GBD 2015 Neurological Disorders Collaborator Group. Global, regional, and national burden of neurological disorders during 1990-2015: a systematic analysis for the Global Burden of Disease Study 2015. Lancet Neurol. 2017; 16:877-97.

2. Jankovic J. Parkinson's disease: clinical features and diagnosis. J Neurol Neurosurg Psychiatry. 2008;79:368-76.

3. Cannon JR, Greenamyre JT. Gene-environment interactions in Parkinson's disease: specific evidence in humans and mammalian models. Neurobiol Dis. 2013;57. https://doi.org/10.1016/j.nbd.2012.06.025.

4. Chin-Chan M, Navarro-Yepes J, Quintanilla-Vega B. Environmental pollutants as risk factors for neurodegenerative disorders: Alzheimer and Parkinson diseases. Front Cell Neurosci. 2015;9. https://doi.org/10.3389/fncel.2015. 00124.

5. Pringsheim T, Jette N, Frolkis A, Steeves TDL. The prevalence of Parkinson's disease: a systematic review and meta-analysis. Mov Disord Off J Mov Disord Soc. 2014:29:1583-90.

6. Lekoubou A, Echouffo-Tcheugui JB, Kengne AP. Epidemiology of neurodegenerative diseases in sub-Saharan Africa: a systematic review. BMC Public Health. 2014;14:653.

7. Williams U, Bandmann O, Walker R. Parkinson's disease in sub-Saharan Africa: a review of epidemiology, genetics and access to care. J Mov Disord. 2018;11:53-64

8. Klein C, Westenberger A. Genetics of Parkinson's disease. Cold Spring Harb Perspect Med. 2012;2. https://doi.org/10.1101/cshperspect.a008888.

9. Lill CM. Genetics of Parkinson's disease. Mol Cell Probes. 2016;30:386-96.

10. Polito L, Greco A, Seripa D. Genetic profile, environmental exposure, and their interaction in Parkinson's disease. Park Dis. 2016;2016. https://doi.org/ 10.1155/2016/6465793.

11. Zschiedrich K, König IR, Brüggemann N, Kock N, Kasten M, Leenders KL, et al. MDR1 variants and risk of Parkinson disease. Association with pesticide exposure? J Neurol. 2009;256:115-20.

12. Jain S, Wood NW, Healy DG. Molecular genetic pathways in Parkinson's disease: a review. Clin Sci Lond Engl 1979. 2005;109:355-64.

13. Clarimón J, Kulisevsky J. Parkinson's disease: from genetics to clinical practice. Curr Genomics. 2013;14:560-7.

14. Popescu C. Mechanisms implicated in Parkinson disease from genetic perspective. Med Clin Rev. 2016;2. https://doi.org/10.21767/2471-299X. 1000028.

15. Blanckenberg J, Bardien S, Glanzmann B, Okubadejo NU, Carr JA. The prevalence and genetics of Parkinson's disease in sub-Saharan Africans. Neurol Sci. 2013;335:22-5.
16. Haylett WL, Keyser RJ, du Plessis MC, van der Merwe C, Blanckenberg J, Lombard D, et al. Mutations in the parkin gene are a minor cause of Parkinson's disease in the south African population. Parkinsonism Relat Disord. 2012;18:89-92.

17. Blanckenberg J, Ntsapi C, Carr JA, Bardien S. EIF4G1 R1205H and VPS35 D620N mutations are rare in Parkinson's disease from South Africa. Neurobiol Aging. 2014;35:445.e1-3.

18. Okubadejo N, Britton A, Crews C, Akinyemi R, Hardy J, Singleton A, et al. Analysis of Nigerians with apparently sporadic Parkinson disease for mutations in LRRK2, PRKN and ATXN3. PLoS One. 2008:3:e3421.

19. Gurdasani D, Carstensen T, Tekola-Ayele F, Pagani L, Tachmazidou I, Hatzikotoulas $\mathrm{K}$, et al. The African genome variation project shapes medical genetics in Africa. Nature. 2015;517:327.

20. Olgiati S, Quadri M, Bonifati V. Genetics of movement disorders in the next-generation sequencing era. Mov Disord Off J Mov Disord Soc. 2016;31:458-70

21. Farlow JL, Robak LA, Hetrick K, Bowling K, Boerwinkle E, Coban-Akdemir ZH, et al. Whole-exome sequencing in familial Parkinson disease. JAMA Neurol. 2016;73:68-75.

22. Steele JC, Guella I, Szu-Tu C, Lin MK, Thompson C, Evans DM, et al. Defining neurodegeneration on Guam by targeted genomic sequencing. Ann Neurol. 2015;77:458-68.

23. Gorostidi A, Martí-Massó JF, Bergareche A, Rodríguez-Oroz MC, López de Munain A, Ruiz-Martínez J. Genetic mutation analysis of Parkinson's disease patients using multigene next-generation sequencing panels. Mol Diagn Ther. 2016;20:481-91.

24. R Core Team. R: A language and environment for statistical computing. Vienna: R Foundation for Statistical Computing; 2018. (https://www.Rproject.org/). https://www.blopig.com/blog/2013/07/citing-r-packages-inyour-thesispaperassignments/. Accessed 5 Oct 2018

25. Liu X, Wu C, Li C, Boerwinkle E. dbNSFP v3.0: a one-stop database of functional predictions and annotations for human nonsynonymous and splice-site SNVs. Hum Mutat. 2016;37:235-41.

26. Kouranov A, Xie L, de la Cruz J, Chen L, Westbrook J, Bourne PE, et al. The RCSB PDB information portal for structural genomics. Nucleic Acids Res. 2006;34(Database issue):D302-5.

27. Kelley LA, Mezulis S, Yates CM, Wass MN, Sternberg MJE. The Phyre2 web portal for protein modeling, prediction and analysis. Nat Protoc. 2015;10:845-58.

28. Toyoshima C. How Ca2+-ATPase pumps ions across the sarcoplasmic reticulum membrane. Biochim Biophys Acta. 2009;1793:941-6.

29. Toyoshima C, Cornelius F. New crystal structures of PII-type ATPases: excitement continues. Curr Opin Struct Biol. 2013;23:507-14.

30. Toyoshima C, Mizutani T. Crystal structure of the calcium pump with a bound ATP analogue. Nature. 2004;430:529-35.

31. van Veen S, Sørensen DM, Holemans T, Holen HW, Palmgren MG, Vangheluwe P. Cellular function and pathological role of ATP13A2 and related P-type transport ATPases in Parkinson's disease and other neurological disorders. Front Mol Neurosci. 2014;7. https://doi.org/10.3389/ fnmol.2014.00048.

32. Karczewski K, Francioli LC, Tiao G, Cummings BB, Alföldi J, Wang Q, et al. Variation across 141,456 human exomes and genomes reveals the spectrum of loss-of-function intolerance across human protein-coding genes. bioRxiv. 2019:531210. https://doi.org/10.1101/531210

33. Kong SMY, Chan BKK, Park J-S, Hill KJ, Aitken JB, Cottle L, et al. Parkinson's disease-linked human PARK9/ATP13A2 maintains zinc homeostasis and promotes a-Synuclein externalization via exosomes. Hum Mol Genet. 2014; 23:2816-33.

34. Monnot S, Serre V, Chadefaux-Vekemans B, Aupetit J, Romano S, De Lonlay $P$, et al. Structural insights on pathogenic effects of novel mutations causing pyruvate carboxylase deficiency. Hum Mutat. 2009;30:734-40.

35. Crino PB, Nathanson KL, Henske EP. The tuberous sclerosis complex. N Engl Med. 2006;355:1345-56.

36. Sowers LP, Loo L, Wu Y, Campbell E, Ulrich JD, Wu S, et al. Disruption of the non-canonical Wnt gene PRICKLE2 leads to autism-like behaviors with evidence for hippocampal synaptic dysfunction. Mol Psychiatry. 2013;18: 1077-89 Note: Erratum: Molec. Psychiat. 19: 742.

37. Filocamo M, Morrone A. Lysosomal storage disorders: molecular basis and laboratory testing. Hum Genomics. 2011;5:156-69.

38. Mokaya J, Gray WK, Carr J. Beliefs, knowledge and attitudes towards Parkinson's disease among a Xhosa speaking black population in South Africa: a cross-sectional study. Parkinsonism Relat Disord. 2017;41:51-7. 
39. Rotimi CN, Bentley AR, Doumatey AP, Chen G, Shriner D, Adeyemo A. The genomic landscape of African populations in health and disease. Hum Mo Genet. 2017;26:R225-36.

\section{Publisher's Note}

Springer Nature remains neutral with regard to jurisdictional claims in published maps and institutional affiliations.

Ready to submit your research? Choose BMC and benefit from:

- fast, convenient online submission

- thorough peer review by experienced researchers in your field

- rapid publication on acceptance

- support for research data, including large and complex data types

- gold Open Access which fosters wider collaboration and increased citations

- maximum visibility for your research: over $100 \mathrm{M}$ website views per year

At $\mathrm{BMC}$, research is always in progress. 